\title{
Resuscitation on the K-1 Yongquan: Ethical and Methodological Aspects of Its Pilot Study
}

\author{
Adrián Ángel Inchauspe \\ School of Medical Sciences, National University of La Plata, Argentina \\ Email: adrian.inchauspe@yahoo.com.ar
}

Received 12 May 2015; accepted 3 July 2015; published 7 July 2015

Copyright (C) 2015 by author and Scientific Research Publishing Inc.

This work is licensed under the Creative Commons Attribution International License (CC BY). http://creativecommons.org/licenses/by/4.0/

(c) (i) Open Access

\section{Abstract}

K-1 Yongquan complementary resuscitation maneuver, systematized since 1987, has been consistently performed in sudden death and cardiac arrest conditions as a final resource in both basic and advanced CPR failure. Experimental analytical studies identify the prevention, control and assessment of treatments set up as well as the determination of their efficiency offering; in that way, there are more possibilities than risks during the trial period. That type of study refers to the random criterion in order to measure differences and, in that way, it establishes causal associations which may better determine the intervention mechanism. As inferred by this criterion, control groups would not profit from the benefit of providing a second chance by means of the proposed maneuver during rescue. Such determination leaves those included in the control group deserted to their own ill-fate, adding - consequently-a certain lethal risk, which should basic and advanced CPR fail. In view of this panorama, we tried to find a methodology that should ensure the validation process according to the model presented; to that end, we propose the Cohort Retrospective Study. All of the above has given evidence that there is a difference if one takes into consideration as control the group of "deceased patients" instead of considering among them "patients that may be deceased". Such apparently simple consideration-thanks to the Cohort Retrospective model-manages to efface the high possibility of a "fatal damage", as proposed by the randomness principle upon a prospective non-intervention group. Beyond the scientific methodology that supports it the efficiency of the maneuver derives mainly from the sustained increase in survival rates presented in the successive statistics published since its application.

\section{Keywords}

Yongquan Maneuver, Pilot-Study, Randomness Analysis, Semiotic Paradigm, Cohort Retrospective Model, Fatal Risk Contingenciality 


\section{Introduction}

The maneuver on acupuncture point K-1 Yongquan has been systematized since 1987 to be proposed as supplementary practice in conditions such as sudden death, cardiac arrest and eventually as a final resource when faced to the failure of basic and advanced CPR [1] [2].

Initially published by the renowned journal Resuscitation [1], it has little by little justified its validity through an increased personal casuistry conformed along almost three decades and several publications [1] [2]; consequently by a most significant statistics: that of adding up survivors to such critical and life impending situation $[2]$.

\section{Stating the Problem}

Experimental analytical studies precisely identify the prevention, control and assessment of the treatments that have been set up as well as the determination of their efficiency thus offering more possibilities than risks during the trial period.

Initially, a methodological design was proposed during investigation in which a certain group would receive the treatment and another would not be benefited (control group). Such type of study makes reference to the random criterion in order to measure differences and, in that way, establish causal associations which may better determine the intervention mechanism [3] [4].

As inferred by the criterion, the control group would not profit from the benefit of providing a second chance by means of the proposed maneuver during rescue. Such determination leaves those included in the control group deserted to their own ill-fate should basic and advanced CPR fail.

Having consulted on the specific issue with Prof. Carlo Guinzburg-Emeritus Professor at Bologna University and a champion of the Index Paradigm - and then submitted a proposal as regards the subjectivity of randomness in scientific research, a research protocol was presented to the authorities of the Joint Committee on Health Research and the Central Ethics Committee of the Province of Buenos Aires - the highest authorities in our region-to be approved.

We would like now to state how the readily available tools of observation and randomness loom over our proposed work from hypothesis to its confirmation; i.e. from our presuppositions to their assertion.

\section{An Analysis of Causation and Randomness}

Both Causation - understood as "presence of a cause" — as Randomness — understood as “absence of certainty"-constitute antagonistic scientific principles which, like Oriental cosmological opposites, find their origin at the extreme of their respective adversary. In fact, between mere chance and absolute certainty, there exist a wide range of shades; one can argue about the most probable cause (i.e. probability) and all the set of events as possible effects [3] [4].

In order to carry out observational studies, it obviously means that observation plays the role of a "magnifier" enabling one to come up with details of what is under observation. However, this instrument is never quite independent from the eye through which a wider view on the causes of the phenomenon under analysis is regarded [3] [4].

As far as experimental studies are concerned, randomness constitutes an optimum objectivity condition that is able to ensure scientific neutrality, deprived of interest or passion on the part of the scientist [4].

In that order, then, we shall review in the following sections, the main speculations assignable to the use of said resources and their consequences as regards research process validation.

\section{Materials}

\subsection{Materials (A)}

\subsubsection{Historical Background about the Methodology of Maneuver Application}

Several authors have defined epistemology as the theory of scientific knowledge; besides, in its original meaning, it refers to that branch of knowledge involved in scientific rules and methods: a complex domain about how one thinks and decides "with expertise and dexterity” (epísteme). Somehow, according to Gustavo A. Apreda, the epistemological problem is concerned with delimitation of languages as well as scientific criteria and methodologies from those which are not [5]. 
The emergence of Science-and its characteristics as known today-dates back to the 17th century, as from the so-called "Copernican revolution". The heliocentric theory led, for example, to the independence of Astronomy and Physics from Physiology, very much like telescopic observations led Galileo Galilei (1564-1642) to break away from the common held idea at the time of the Earth being the center of the Universe.

Bearing a physiological as well as an epistemological perspective in mind, Galileo studied Chaos in the Universe (and the complexity of its apparent causation) associating it to man's incapacity to pay attention to every event within a concrete space and a determined time, assuming, according to Apreda "the uncertain conceptualization of fate: what is undetermined... uncertainty... randomness... as opposed to the order Logos” [6].

Such "New Knowledge Order" shall find-according to renowned contemporary Italian historian Carlo Guinzburg - an emerging counterpoint towards the end of the 19th century, with the "Semiotic or Index Paradigm”, an epistemological model that confronts the traditional, Galilean one employed in Physics.

\subsection{2. "The Index Paradigm: Genesis and Development" [7]}

An article was published by the Faculty of Social Sciences at the University of Chile by Argentinian authors Pulice, Manson and Zelis under the title above. In that article these authors make reference to Prof. Guinzburg when he stated how “...thus, silently, there emerged, within the area of social sciences, an epistemological model (or, if preferred, a paradigm). The analysis of said paradigm, which has not to date received the attention it deserves and has been in use even when its theory has not been yet explicitly formulated, may perhaps contribute to overcome the sterile opposition between 'rationalism' and 'irrationalism'” [7].

The following chart accounts for Guinzburg's counterpoint between the Index Paradigm and that of Galilean Physics (Figure 1), the latter being_according to the Italian historian-the beginning of modern Physics [7]. The oppositions find clear evidence in the highlighted points (see again Figure 1).

According to Guinzburg, at the root of this paradigm is the remote period in which human beings lived out of hunting, reconstructing the appearance and the movements of an invisible prey through the analysis of traces or clues. Learning to observe, they gave significance even to the smallest print within a determined context. Successive generations of hunters could thus transmit their experience of knowledge, one which Guinzburg has called "hunting knowledge" [7].

There is a link between the first symbolic articulations man made in order to be able to predict events ("magical thinking”) and what we can today call "suppositions”. Guinzburg states that what is inferred there are the possibilities, not the necessary consequences or verifiable events. Such approach implies the analysis of particular cases, which could only be reconstructed by means of prints, symptoms or clues. [7]

One is able to foresee, in some references dealing with the practice of Medicine in Ancient Greece-which can be found in the texts that constitute the Hippocratic Corpus (between 5th and 6th centuries B. C.) - how that paradigm set the bases for a long-standing "medical semiology" which took very long to be surpassed. According to Guinzburg, the index model was totally eclipsed by the more prestigious Platonic theory of knowledge and has survived to these days merely in an implicit form [7].

\subsection{Materials (B): K-1 Yongquan Theory through Acupuncture Principles [8]}

Through the Ling Shu analysis (chap.5), K-1 is also hierarched as the "root" point of the Shao Yin. This energetical level, formed by the Kidneys and the Heart. Moreover, Yongquan is the main place for the ascending Yin

\begin{tabular}{|c|c|}
\hline \multicolumn{2}{|c|}{ RESEARCH PARADIGM OPPOSITION } \\
\hline GALILEAN PHYSICS' PARADIGM & INDEX PARADIGM \\
\hline $\begin{array}{c}\text { Gives priority to what can be repeated, } \\
\text { measured, communicated; to } \\
\text { generalizations; to coincidences }\end{array}$ & $\begin{array}{c}\text { Gives priority to what cannot be } \\
\text { repeated; towhat is singular; to an } \\
\text { original thing; to what is surprising }\end{array}$ \\
\hline Quantitative research & Qualitative research \\
\hline $\begin{array}{c}\text { Shows interest in universals, in rules; } \\
\text { discardsindividual characteristics }\end{array}$ & $\begin{array}{c}\text { Interestin what is individual, in a } \\
\text { particular case }\end{array}$ \\
\hline Studies that which is typical & Studiesthat which is exceptional \\
\hline
\end{tabular}

Figure 1. Pulice, G.; Manson, F.; Zelis, O. "The Indiciary Paradigm: Genesis and Deployment” [7]. 
Qi from the Earth into our body. Therefore, this kind of energy will nurture the zhang, especially those placed in the most Yang (or higher) part of the torso, in order to interact with the Heavenly energy, essential a non-interruptable function to maintain our life, like the heart and the lungs. It can be read in Ling Shu, chap 9: "Yin rules the organs, while Yang rules the viscus. Yin absorbs the Heavenly energy, while Yang absorbs the energy from the Five Organs". Thus, the Celestial Yang Qi provides them with continuous motility in the Upper Jiao of our body, for a perfect vital equilibrium [8].

\subsection{Materials(C): Maneuver on Acupuncture K-1 Yongquan Point}

Even though it constitutes a supplementary methodology to add to PCR protocols, in this article the maneuver is proposed as the material upon which ethical, statistical and scientific models shall be analyzed, and which shall eventually validate data and conclusions.

The application of the maneuver is really simple: in a prone/supine position-no specific position is required, strong pressure is exerted on the point mentioned above by means of digital pressure (preferably, the thumb) or a common object (such as a toothbrush or a pen) at the union between $1 / 3$ anterior and $2 / 3$ posterior of the sole; at the gap that is produced when the sole flexes. Then the maneuver is carried out at 3 - 5 second stimuli until it proves positive (i.e. cardiac activity is reinitiated as evidenced by ECG and pulse) [1].

\subsection{Materials (4): Indications of the Yongquan Maneuver Integrated to ILCOR's PCR Protocol}

The author of this paper has-to date-presented several works and conferences dealing with the global situation of statistics on mortality due to cardiac arrest and sudden death in a number of countries [9], that in accordance with the WHO forecast as regards deaths due to cardiovascular cause (both in the so-called countries of the First World and those of Developing Countries) [10]. To those, an additional percentage should be added which derives from cerebrovascular causes of death, for the same Yongquan maneuver also contributes to the recovery from potential brain damage after a stroke [11]. A stroke, it needs to be underlined, may be considered both cause as well as consequence of cardiac arrest; that is why it seems only right to add these statistical figures to cardiovascular deaths. According to the WHO, the percentage of these additions reaches almost $30 \%$ of all the general causes of death on the planet [10].

The maneuver can be implemented on persons within cars that have collided or are overturned; it also can be useful in situations of landslip or building collapse, even when those affected are masses. Beyond being suitable and able to be integrated along each and any of the "action chain" listed in the ILCOR's CPR Protocol, it becomes a "Golden Standard” when faced with failure in basic and advanced CPR [2] [10]

\section{Methods: Proposing the Semiotic or Index Paradigm in a Pilot Study on Yongquan}

\subsection{The Constitution of a Random Event}

A random event is generally defined by three characteristics: futurity, incertitude and contingentiality.

Futurity: it implies that a random event is something that can take place in the future; i.e., it is not actually occurring but it is merely potential.

Incertitude: (oruncertainty) refers to the lack of certainty; an intermediate state between necessity (certainty that something is to take place) and impossibility (utter exclusion of it taking place). Incertitude as such can be configured in gradual categories, namely:

1) Certain, but uncertain

2) Uncertain, but probable

3) Improbable, but not impossible

4) Possible, butunpredictable

Contingentiality: it includes risk as an essential element in the random process, considering as such " $a$ preexistent reality or possibility of suffering damage" which implies the consequences of confronting its transfer.

Along that line, it would be convenient—so as to characterize the random event — to replace the term risk (as possibility of suffering any detriment) in favor of a more neutral one, that of contingency, which denotes "the possibility of something happening or not" which, besides, defines itself as "uncertain future” [12]. 


\subsection{Importance of the Random Event}

In terms of the Four Fundamental Principles in Ethics [13], the concept of "random event" acquires a double meaning:

a positive one, related to the idea of gain or benefit

$$
\text { (Beneficiency) }
$$

a negative one, implying the possibility of loss, adversity or damage

(Malediciency)

The key to the success in considering both positions lies, to a great extent, in argumentation understood as the timely management of the different resources available, an increased degree of knowledge, and an enhanced skill in using them wisely.

\subsection{Application of Statistical Analysis Based on the Index Paradigm [2] [14]}

As we shall see in the Discussion section below, the randomness principle requires an ever increasing sample-following a quantitative interest—in order to get away from uncertainty, thus reducing the variability of fate. Being generally the case in the validation of any statistical methodology applied in Medicine, it has to make an effort to prove if any association visible through observation is a product of fate or not [2] [14].

Given the fact that one of the main differences between Galilean and Semiotic Paradigms is that the former strives to find deductions from the general to the particular case, the latter gives emphasis to that which is individual, original and qualitative, we tried to find a methodology that should ensure the validation process according to the model presented. To that end, we proposed the Cohort Retrospective Study [15] assisted by engineers Silvia Roggero and Sergio Fernández, whose invaluable contribution we highly appreciate.

In spite of what has been so far stated, understanding that we might not eventually be able to solve every single question, we have given priority to what in our view is singular and of qualitative nature. As such, we have been able to prove the following affirmation proposed between two hypotheses:

Ho (null hypothesis): its affirmation determines the lack of association between the variables under study;

Ha (alternative hypothesis): its affirmation implies some degree of relationship between the variables.

Statistical analysis shall assist our selection of the correct option by observing the breach made manifest between both treatments: i.e., differences between control group (those who died without the Yongquan maneuver treatment) and those who received the benefit of the maneuver. Should the difference be higher than standard error multiplied by a safety coefficient, we shall then be led to conclude that there in fact exists a significant difference between both variables: that means that Ha shall be accepted and, consequently, Ho, rejected [2].

What we first did was to compare the group who were assisted by CPR maneuvers without the use of a defibrillator (method "A") and those assisted by Yongquan resuscitation (method "B"). This was carried out by using percentages of representative samples:

- Method "A" treatment: $6.5 \%$ response

- Method "B" treatment: $84.84 \%$ response

If the difference [PA - PB] (where P is probability; and A and B are the analyzed methods) is higher than the product; that is, 1.96 times the standard error (SE), the difference shall then be significant.

$|\mathrm{PA}-\mathrm{PB}|=|0.064-0.85|=0.786<\mathrm{SE}(0.05) \times 1.96=0.098$

Because $[\mathrm{PA}-\mathrm{PB}]=0.786$ is higher than 0.0098 , the difference between 0.064 and 0.85 is, actually, statistically significant.

We are, then, entitled by theoretical foundations to reject Ho hypothesis and accept Ha hypothesis as correct. The aforementioned implies that FATE MAY BEIMPROBABLE RESPONSIBLE of that association.

This analysis, we believe, definitively proves the K-1 Yongquan resuscitation method bears a "quality guarantee" [2].

Afterwards, we compared the use of defibrillation (Method "A") against the supplementary Yongquan resuscitation maneuver (Method "B"). Afterlife indexes on both treatments were as follows:

- Method "A" treatment: $48 \%$ response

- Method "B" treatment: $84.84 \%$ response

$|\mathrm{PA}-\mathrm{PB}|=|0.48-0.84|=0.36<\mathrm{SE}(0.0076) \times 1.96=0.0148)$.

Thus, $\left[\mathrm{PA}^{\prime}-\mathrm{PB}\right]=0.36$.

Because this result is also higher than standard error, (SE) multiplied by 1.96, it provides a value of 0.00148 , 
a figure which also proves to be statistically significant. This, again, proves the statistical value of the supplementary resuscitation K-1 Yongquan point by means of comparative analysis

\subsection{Progression of the Presented Statistics}

This progression was introduced as Section C Poster Presentation ( $\left.\mathrm{N}^{\circ} 0100\right)$ in the $9^{\text {th }}$ International Brain Injury Association Congress [9]—Edinburgh, March 23-26, 2012.

First statistic: November 2005

$50^{\text {th }}$ Argentina Acupuncture Society Congress 19 patients-5 deaths

Survival Rate: $73.68 \%$

> Second statistic: November 2009

Lazarus Effect Video 27 cases-7 deaths.

Survival Rate: $74.07 \%$

> Third statistic: January 2010

Resuscitation Journal [1] 30 cases—8 deaths

Survival Rate:73.33\%

Fourth statistic:

$8^{\text {th }}$ IDDST Congress, Beijing 39 cases-8 deaths

Survival Rate:79.48\%.

$>$ Fifth statistic:

In Tech's Publication [8] $\quad 44$ cases -9 deaths

"Recent Advances in Theories and Practice in Chinese Medicine" [8]

Survival Rate:79,53\%

$>$ Sixth statistic:

$9^{\text {th }}$ International Brain Injury Congress [11] 52 cases—9 deaths

Survival Rate: $82.69 \%$

>Seventh statistic: $84.48 \%$

World Journal of Critical Care Medicine, August $4^{\text {th }}$, 2013 [2] 58 cases-9 deaths

See survival statistics' tendencies in Figure 2.

Consequently, all the above has been made clear that there actually is a difference if one takes into account as control the group of "deceased patients" instead of considering among them "patients that may be deceased". Such apparently simple consideration — thanks to the Cohort Retrospective Study model [15]—manages to efface the contingentiality of a possible "fatal damage" as proposed by the randomness principle upon a prospective non-intervention group.

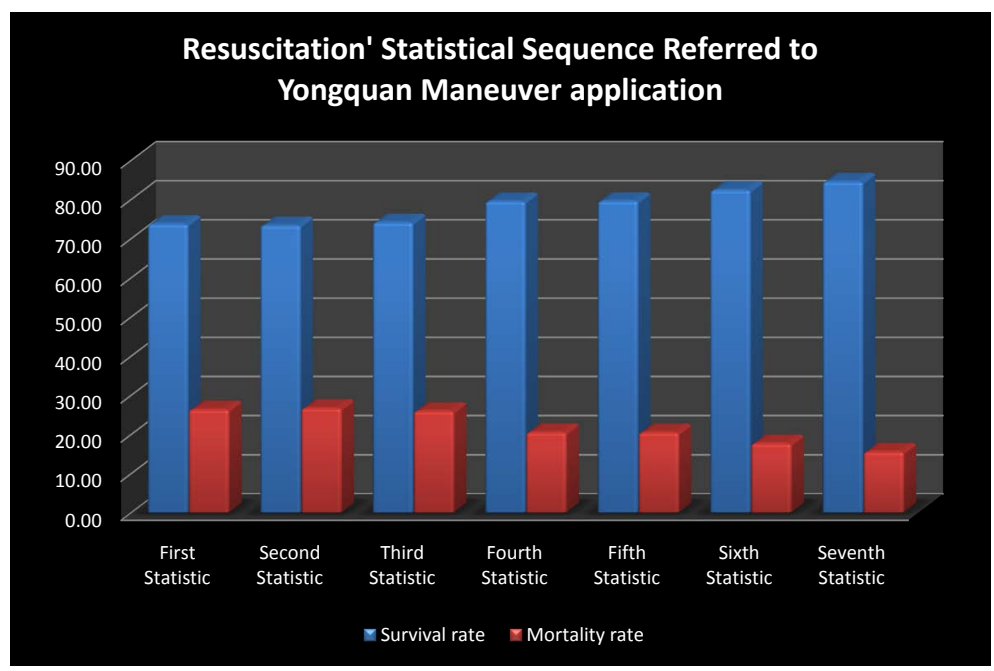

Figure 2. Resucitation' Statistical Sequence Referred to Yongquan Maneuver application-Inchauspe A.A. in "Guideline of Application of resuscitation maneuver on the acupuncture point R-1 Yongquan”. 


\subsection{The Value of Casuistry Resulting from Its Application on Yongquan Maneuver}

Going beyond the scientific methodology that supports it-both in principles of TCM as in its inclusion in animal rescue and Uttstein Style protocol comparative analysis-the efficiency of the maneuver derives mainly from the sustained increase in survivalrates presented in the successive statistics published since its application $[2]$.

According to what we previously considered when dealing with its indications, the maneuver on Yongquan may adequately integrate as supplementary resuscitation maneuver to current ILCOR's protocol of basic and advanced resuscitation valid worldwide. This inclusion would in no way infringe any of the gestures making up its "action chain" [2].

Because it constitutes a unique variable, the control group can be made up of all those persons deceased previous to the protocol inclusion of the maneuver; that, in other words, would amount to the group of victims and statistical percentages and survival rates previous to the implementation of this therapeutic resource, eliminating in this way the impairment found in random contingentiality, which in this study in particular, would result in a serious-fatal-loss mentioned in the sections above.

\section{Discussion}

\subsection{General Disadvantages of Randomness}

During a course we took in 2013, [Official Course in Ethics and Health Research. Province of de Buenos Aires] three "Misfortunes" were mentioned in the process of evaluation data provided by research: random mistake, bias, and mistakes originated in confusion.

In the first case, in order to alleviate the random mistake, a suggestion was made as to solving the uncertainty brought about by fate by increasing the number of the sample population; i.e. to expand the number of people under investigation (a set of an area of analytical interest).

As regards bias, another solution was proposed based on the already available rules to reject the null hypothesis: the level of statistical signification by means of pertinent tests: namely, Arithmetic mean and standard deviation, also known as "random variables".

In as far as confusion is concerned, the risk of an independent exhibition of results was alleviated by a specific modality of clinical trial that we shall consider further ahead: stratification.

\subsection{New, Specific Disadvantages to Randomness}

As regards Randomness Maleficiency, this would truly constitute-in the supposed case of suggesting a control group for this pilot study — a lethal consequence: i.e., purposefully depriving a patient in an imminent death situation from an opportunity of survival [1].

As was shown when stating the problem above, the control group would not only not benefit from a second chance of survival during such severe imminent death situation, but also such therapeutic discrimination would also imply a fatal, collateral and unwanted result for the members of that group, they are doomed.

This consequence is rooted in the three characteristics that are associated to the random process, namely:

-The prospective condition of Futurity, which required the consideration of a non-application group within the study in question;

-The impossibility of Contingentiality in transferring or replacing risk and;

-The possibility of suffering harm becomes the source of Uncertainty towards the future of the proof.

The situation presented here establishes an exception that should be regarded as worth being taken into consideration during the stage of planning an adequate scientific methodology in order to assess these practices under conditions that are similar to those drawn here.

\subsection{Solutions to the Randomness Problem}

\subsubsection{Solution $\mathrm{N}^{\circ}$ 1: Types of Sample}

The sampling theory aims at establishing the procedures through which conclusions about a certain population can be drawn starting from a sub-group within it with a minimal error margin.

As randomness requires listing all the characteristics of the units that make up a population, it supposes a 
bigger simple size than those of other designs in order to attain the same reliability level.

A stratified sampling was suggested as a plausible way out for dealing with the confusion variable, for it may result convenient to divide the population under study into categories or strata with an analytical interest as regards the argumentation of the work, in order to obtain greater homogenization of the final sample [12].

Even though stratification guarantees representation and provides more information about small groups, it often presents difficulties when in the very process of determining strata. That is why the decision to start an initial phase with a pilot study was taken in which simple needs not be stratified and might envision a first approach to results before suggesting a multicenter study.

\subsubsection{Solution $\mathrm{N}^{\circ}$ 2: Considering the Semiotic Paradigm as Possible Scientific Validation Modality}

That knowledge is acquired by a subject who is the active agent of the action goes beyond the mere conception of "that which is objective" as the real power to know the reality of the world and "that which is subjective" as the experience of knowledge in the subject in order to reach the truth. When capturing reality, we actually "perceive" it. Failing to acknowledge this distinction, according to Descartes, constitutes a source of error which is constant in the scientific process [16].

The Semiotic Paradigm turned out to be, in this particular case, another possible solution to the serious consequences randomness sets out, for-based on the observation of a single, unique phenomenon —it proposes a plausible scientific outcome. Such solution is based on very solid ground, for it is the first rule Descartes stated in his Discourse Method: Evidence/Proof.

Very much like one finds it quite simple to demonstrate that every hypothesis (i.e., aproposal deriving, most often, from processes of construction by trial and error) may be established as the beginning for scientific process to start, also Evidence (according to Descartes, first rule of the book mentioned above, equivalent to the Truth criterion [17] can also set into movement the whole scientific sequence.

The latter was made visible when a statistical signification was achieved by using a Cohort Retrospective Study model with metachronic inclusion criterion for the affected groups, knowing beforehand the potential problems that may take place in the control group. Somehow, the effects derived from the main characteristics of randomness which gave way to deadliness were reverted:

-First, Futurity was modified by searching for a control group in the past, in that way no future samples would be at risk of possible death due to non-intervention on that part of the studied population.

-Secondly, should the foregoing clause remain unmodified, the case might be that Contingentiality - rendered unable to transfer the risk typical of the random process - would be completely distorted tragically affecting the non-intervention group within the study.

-As a result, Uncertainty was drastically reduced for individuals from the control group were in no danger while being subjected to a comparative analysis with those receiving the supplementary resuscitation maneuver.

Taking into consideration the moral, bioethical and legal aspects of its application, this paper represents an exceptional case, for the randomness principle which provides the foundation to current global scientific methodology would severely damage the control group under study. Random non-intervention practiced on such a group would inevitably lead to a most certain "Russian Roulette" effect as well as a most serious ethical problem: not providing the necessary and timely assistance that may save a life to those patients who had been "sorted out", i.e. destined not to be benefitted by the application of the maneuver.

Resorting to metachronic groups of sudden death and cardiac arrest victims, randomness unfavorability was solved reaching in that sense a $P$ significance totally valid as product of a correct statistical analysis.

Article 32 of the Declaration of Helsinki VI on Ethical Principles for Medical Research Involving Human Subjects and Human Rights should not be forgotten when it states:

32. In the treatment of a patient, where proven prophylactic, diagnostic and therapeutic methods do not exist or have been ineffective, the physician, with informed consent from the patient, must be free to use unproven or new prophylactic, diagnostic and therapeutic measures, if in the physician's judgement it offers hope of saving life, reestablishing health or alleviating suffering. Where possible, these measures should be made the object of research, designed to evaluate their safety and efficacy. In all cases, new information should be recorded and, where appropriate, published (http://www.wma.net/es/30publications/10policies/b3/17c_es.pdf)

Art. $32^{\circ}$ Helsinski VI, in essence proposes a "Research Ethics", one that goes beyond statistics: if a single life out of a million can be saved, then, the maneuver shall find its justification. This represents the epitome of the Index Paradigm: putting value on the impact of that which is particular with respect to a general result (Apreda, 
pers. comm.).

A physician should, then, use every possible means available to better cater for the patient in such extreme conditions as imminent death, an extremely urgent situation [18].

\section{Conclusions}

The core problem of observation remains, undoubtedly, the observer; for it is he who needs to assimilate the information derived from his observations in order to arrive at conclusions that may support his hypothetical constructions.

Beyond providing this technique with a most rigorous objectivity, the main consideration in any observation shall be to accurately determine that which is to be observed; that is, to precisely define in an unambiguous way, the support of this observation.

That is why the main task in the actual practice of observation shall be to assign exhaustive categories in order to define the universe to be observed as well as the adequate statistical instrument for each particular study situation.

A traditional current, objectivism, excludes the observer from observation. In Apreda's terms, his "theoretical assumptions" contribute to stating the hypothesis to be contrasted or rejected, paving the way for the hypothetical-deductive path [6].

Maybe this led was what led Karl Popper to prove back in 1930 that "scientific proof is never conclusive": but just a new piece of data which can either rectify or reject what has been previously held. Thus, we can only arrive at partial confirmations: in other words, a probability [19].

Thus, for Bateson, the validation of observational experience "shall depend on the basic criteria accepted for such arguments", for reality binds observer and what is being observed from a conceptual perspective [20].

Consequently, premises of the scientific method shall invariably be dependent on the flexibility of the researcher's criteria, researcher who can never be considered alien to the validation of his own experience.

Randomness is associated to every process the result of which is unpredictable; that means it can never be determined before it is produced. That is why the study of random phenomena belongs to the sphere of probability and, in general, to the statistics theory, correlating the different measurable statistical properties [6]. What has previously been stated contrasts with the very essence of that principle, for data are previously known about the fatal contingency which randomly discarded patients are bound to suffer, for they shall be deprived of the maneuver in case of basic and advanced CPR should they belong to control group. The ethical and moral dimensions of such decision translate in a most severe offence: that of a patient being abandoned.

As previously stated, such apparent set of initial conditions-theoretically resulting in an unpredictable and unrepeatable experience-may lead to quite different results... as if "throwing dices". However, taking into consideration not only events in the future but also the good decisions of the past, seems to offer a determining phenomenon in the evolution of statistical methodology [11].

In this way, the contradiction in between randomness - implying insecurity and uncertainty—and certainty-which presupposes rationality, calculus assessment, etc.- there exists a temporality criterion that enables overcoming such difficulties which, in some cases, configure randomness as something arbitrary, not subjected to rule or measurement. Then, one shall be able to turn to the "alternative hypothesis" in order to achieve in future research a sound and founded impartial virtuous criterion [18].

\section{Final Remarks}

Once the thesis was approved, the research on the maneuver on Yongquan point was submitted to a most detailed methodological during the construction of the protocol under de dedicated supervision of Lic. Norma Domancich. Every requirement for approval being completed, hospital authorities applied for the implementation of a pilot study-under the pre-established condition of not discriminating any victim of sudden death or cardiac arrest during the trial period, thus enabling the possibility of recovery to every patient assisted in such extreme condition. There was only an exceptional comment as regards patients who were admitted to intensive care in abstention or withdrawal of vital support or those with certified brain death. This exception prevents statistical results from including cases which cannot be rescued, notwithstanding that the maneuver may be implemented on every patient.

Authorities from Hospital "San Roque" at Manuel B. Gonnet, La Plata district; the Ethics Committee Coordinator, Dr. Juan Pablo von Arx and the coordinator for the Teaching and Research Committee, Dr. Francisco 
Alberto Cañada, have formally requested to begin the pilot study aimed at confirming the statistical estimation presented to date on 75 treated patients (84.84\% survival) in order to, afterwards, proceed to inviting other medical centers to join in in a multicenter analysis that shall gather a significant number of cases so as to propose the inclusion of the maneuver within current CPR protocols.

\section{Acknowledgements}

I would like to express my gratitude to Amanda B. Zamuner forher most valuable contribution.

\section{References}

[1] Inchauspe, A.A. (2010) Traditional Chinese medicine K-1 Yongquan and Resuscitation: Another Kind of Lazarus Phenomenon. Resuscitation, 81, 505-506. http://dx.doi.org/10.1016/j.resuscitation.2009.12.009

[2] Inchauspe, A.A. (2013) Drawing the Yongquan Protocol into the Different Stages of the Cardiopulmonary Resuscitation Sequence. World Journal of Critical Care Medicine, 2, 17-20.

[3] Apreda, G.A. (2010) Psychopathology, Psychiatry and Mental Health-Its Paradigms and Integration. Editorial of the National University of La Plata, 46.

[4] Apreda, G.A. (2010) Psychopathology, Psychiatry and Mental Health-Its Paradigms and Integration. Editorial of the National University of La Plata, 47

[5] Apreda, G.A. (2010) Psychopathology, Psychiatry and Mental Health—Its Paradigms and Integration. Editorial of the National University of La Plata, 38.

[6] Apreda, G.A. (2010) Psychopathology, Psychiatry and Mental Health-Its Paradigms and Integration. Editorial of the National University of La Plata, 30.

[7] Pulice, G., Manson, F. and Zelis, O. (2001) IV. The Indiciary Paradigm: Genesis and Deployment. In: La Práctica de la Investigación en relación al Pensamiento Mágico, la Conjetura, el Paradigma Indiciario y la Ciencia Moderna. Notas para repensar la cientificidad, Cinta de Moebio. Revista de Epistemología de Ciencias Sociales, No. 12, Chile. http://www.facso.uchile.cl/publicaciones/moebio/12/pulice.htm

[8] Inchauspe, A.A. (2011) Chap. 18. Traditional Chinese Medical Criteria about the Use of Yongquan as a Life-Support Maneuver. In: Kuang, H.X., Ed., Recent Advances in Theories and Practice of Chinese Medicine, InTech Publ. Croatia, 361.

[9] Inchauspe, A.A. (2010) My Experience in Cardiovascular Resuscitation through K-1 Yongquan. VII Congreso de FILASMA-XVII Curso Internacional de Acupuntura, Farmacopea y Medicina Tradicional China, IV Simposium SAME, Seville.

[10] Inchauspe, A.A. (2013) Sudden, Predictable and Preventable Death in Pharmacological Cardio Safety. The 5th International Conference on Drug Discovery and Therapy, Dubai, UAE.

[11] Inchauspe, A.A. (2012) Traditional Chinese and Brain Protection: Can Its Principles Be Consistent with Western Science? Poster Presentation at the 9th IBIA International Brain Injury Association Congress, Edinburgh, 21-25 March 2012.

[12] Mainella, A. and Domancich, N. (2013) Official Course in Ethics and Health Research. Province of Buenos Aires, Argentina.

[13] Montoro Ballesteros, A. (2005) Aleatoriedad en el derecho y el problema de la Justicia (Randomness in Law and the Problem of Justice). Cátedra de Filosofía del Derecho, Anales del Derecho No. 23, University of Murcia, Spain, 71-102.

[14] Apreda, G.A. (2010) Psychopathology, Psychiatry and Mental Health—Its Paradigms and Integration. Editorial of the National University of La Plata, 46.

[15] Kopitowski, K. (2013) Commissioning of Research. Training Program for the Ethics and Research Committees of Buenos Aires Province.

[16] Descartes, R. (1680) Metaphysical Meditations. The Blackwell Guide to Descartes’ Meditations. http://onlinelibrary.wiley.com/doi/10.1002/9780470776476.app1/pdf

[17] Descartes, René. Discourse on Method. Vol. XXXIV, Part 1. The Harvard Classics. P.F. Collier \& Son, New York, 1909-14; Bartleby.com, 2001. http://www.bartleby.com/34/1/2.html/

[18] Von Arx, J.P. (2013) Research Ethics. Plan de Capacitación en Investigación en Salud en la Provincia de Buenos Aires. Hospital de Alta Complejidad “El Cruce”, Florencio Varela.

[19] Popper, K.R. and Eccles, J.C. (1980) The Self and Its Brain. Ed. Labor, Barcelona.

[20] Bateson, G. (1981) Form, Substance and Difference. General Semantics Bulletin, No. 37. 